\title{
On the Periodicity of Some Classes of Systems of Nonlinear Difference Equations
}

\author{
Stevo Stević, ${ }^{1,2}$ Mohammed A. Alghamdi, ${ }^{2}$ Dalal A. Maturi, ${ }^{3}$ and Naseer Shahzad ${ }^{2}$ \\ ${ }^{1}$ Mathematical Institute of the Serbian Academy of Sciences, Knez Mihailova 36/III, 11000 Beograd, Serbia \\ ${ }^{2}$ Department of Mathematics, King Abdulaziz University, P.O. Box 80203, Jeddah 21589, Saudi Arabia \\ ${ }^{3}$ Department of Mathematics, Faculty of Science, King Abdulaziz University, P.O. Box 42641, Jeddah 21551, Saudi Arabia
}

Correspondence should be addressed to Stevo Stević; sstevic@ptt.rs

Received 25 September 2013; Revised 3 December 2013; Accepted 4 December 2013; Published 23 January 2014

Academic Editor: Josef Diblik

Copyright (C) 2014 Stevo Stević et al. This is an open access article distributed under the Creative Commons Attribution License, which permits unrestricted use, distribution, and reproduction in any medium, provided the original work is properly cited.

Some classes of systems of difference equations whose all well-defined solutions are periodic are presented in this note.

\section{Introduction}

There has been a great recent interest in studying difference equations and systems of difference equations which do not stem from differential ones (see, e.g., [1-19] and the references therein). For some results on concrete systems of nonlinear difference equations, see, for example, [1, 3-5, 9-12, 18, 19]. Some classical results in the topic can be found, for example, in book [20].

Solution $\left(x_{n}^{(1)}, \ldots, x_{n}^{(l)}\right)_{n \geq-k}$, of the system of difference equations

$$
\begin{aligned}
x_{n}^{(1)} & =f_{1}\left(x_{n-1}^{(1)}, \ldots, x_{n-k_{1}}^{(1)}, \ldots, x_{n-1}^{(l)}, \ldots, x_{n-k_{l}}^{(l)}\right), \\
x_{n}^{(2)} & =f_{2}\left(x_{n-1}^{(1)}, \ldots, x_{n-k_{1}}^{(1)}, \ldots, x_{n-1}^{(l)}, \ldots, x_{n-k_{l}}^{(l)}\right), \\
& \vdots \\
x_{n}^{(l)} & =f_{l}\left(x_{n-1}^{(1)}, \ldots, x_{n-k_{1}}^{(1)}, \ldots, x_{n-1}^{(l)}, \ldots, x_{n-k_{l}}^{(l)}\right),
\end{aligned}
$$

where $n \in \mathbb{N}_{0}$ and $k=\max \left\{k_{1}, \ldots, k_{l}\right\}$, is called eventually periodic with period $p$, if there is an $n_{1} \geq-k$ such that

$$
x_{n+p}^{(j)}=x_{n}^{(j)},
$$

for every $j=\overline{1, l}$, and $n \geq n_{1}$. It is periodic with period $p$ if $n_{1}=-k$. Period $p$ is prime if there is no $\widehat{p} \in \mathbb{N}$, $\widehat{p}<p$, which is a period. If all well-defined solutions of an equation or a system of difference equations are eventually periodic with the same period, then such an equation or system is called periodic. For some results on the periodicity, asymptotic periodicity and periodic equations or systems of difference equations see, for example, [1-10, 12-14, 16-19] and the related references therein.

In recent paper [19], the authors formulated four results which claim that the following systems of difference equations are periodic with period ten:

$$
\begin{aligned}
& x_{n+1}=\frac{y_{n}}{x_{n-1}\left(1+y_{n}\right)}, \quad y_{n+1}=\frac{x_{n}}{y_{n-1}\left(1+x_{n}\right)}, \quad n \in \mathbb{N}_{0} ; \\
& x_{n+1}=\frac{y_{n}}{x_{n-1}\left(-1+y_{n}\right)}, \quad y_{n+1}=\frac{x_{n}}{y_{n-1}\left(-1+x_{n}\right)}, \quad n \in \mathbb{N}_{0} \text {; } \\
& x_{n+1}=\frac{y_{n}}{x_{n-1}\left(1+y_{n}\right)}, \quad y_{n+1}=\frac{x_{n}}{y_{n-1}\left(-1+x_{n}\right)}, \quad n \in \mathbb{N}_{0} \text {; } \\
& x_{n+1}=\frac{y_{n}}{x_{n-1}\left(-1+y_{n}\right)}, \quad y_{n+1}=\frac{x_{n}}{y_{n-1}\left(1+x_{n}\right)}, \quad n \in \mathbb{N}_{0} \text {. }
\end{aligned}
$$

First, we show that all the results in [19] follow from known ones in the literature and also present some extensions 
of these results in the spirit of systems (3)-(6). To do this, we will use a system of difference equations related to the following, so called, Lyness difference equation:

$$
x_{n+1}=\frac{1+x_{n}}{x_{n-1}}, \quad n \in \mathbb{N}_{0} .
$$

It is easy to see that every well-defined solution of (7) is periodic with period five. The equation arises in frieze patterns (for the original sources, see [21-23]).

Studying max-type equations and systems of difference equations is another topic of a recent interest (see, e.g, [2, 3, 5$7,10,11,15-19])$.

Some special cases of the following max-type difference equation:

$$
x_{n}=\max \left\{\frac{A_{n}}{x_{n-s}}, x_{n-k}\right\}, \quad n \in \mathbb{N}_{0},
$$

where $s, k \in \mathbb{N}$, and $\left(A_{n}\right)_{n \in \mathbb{N}_{0}} \subset \mathbb{R}$, have been studied, for example, in $[2,16]$. Positive solutions of $(8)$ are periodic in many cases. However, if $\left(A_{n}\right)_{n \in \mathbb{N}_{0}}$ is not a positive sequence, it was shown in [2] that (8) can have unbounded solutions.

In [5], it was shown that all solutions of the following max-type system of difference equations:

$$
\begin{array}{r}
x_{n+1}=\max \left\{\frac{A_{n}}{y_{n}}, x_{n-1}\right\}, \quad c y_{n+1}=\max \left\{\frac{B_{n}}{x_{n}}, y_{n-1}\right\}, \\
n \in \mathbb{N}_{0},
\end{array}
$$

where $x_{0}, x_{-1}, y_{0}, y_{-1} \in(0,+\infty)$ and $\left(A_{n}\right)_{n \in \mathbb{N}_{0}},\left(B_{n}\right)_{n \in \mathbb{N}_{0}}$ are positive two-periodic sequences, are eventually periodic with, not necessarily prime, period two. This was done by direct calculation.

By using direct calculation, it can be easily shown that positive solutions of the following max-type system of difference equations:

$$
\begin{array}{r}
x_{n+1}=\max \left\{\frac{A_{n}}{x_{n}}, y_{n-1}\right\}, \quad y_{n+1}=\max \left\{\frac{B_{n}}{y_{n}}, x_{n-1}\right\}, \\
n \in \mathbb{N}_{0},
\end{array}
$$

where $\left(A_{n}\right)_{n \in \mathbb{N}_{0}}$ and $\left(B_{n}\right)_{n \in \mathbb{N}_{0}}$ are positive two-periodic sequences, are also periodic.

Here, we give a noncalculatory explanation of the fact by proving that positive solutions of the following max-type system of difference equations:

$$
\begin{array}{r}
x_{n}=\max \left\{\frac{A_{n}}{x_{n-s}}, y_{n-k}\right\}, \quad y_{n}=\max \left\{\frac{B_{n}}{y_{n-s}}, x_{n-k}\right\}, \\
n \in \mathbb{N}_{0},
\end{array}
$$

where $s, k \in \mathbb{N}$, and $\left(A_{n}\right)_{n \in \mathbb{N}_{0}},\left(B_{n}\right)_{n \in \mathbb{N}_{0}}$ are positive periodic sequences of a certain period, are also periodic. We also present another extension of the result.

\section{Some Extensions of Systems (3)-(6)}

In this section, we present some periodic systems of difference equations in the spirit of systems (3)-(6).

Theorem 1. Consider the following system of difference equations

$$
\begin{aligned}
x_{n+1}^{(1)} & =f_{1}^{-1}\left(\frac{1+f_{2}\left(x_{n}^{(2)}\right)}{f_{3}\left(x_{n-1}^{(3)}\right)}\right), \\
& \vdots \\
x_{n+1}^{(k-1)} & =f_{k-1}^{-1}\left(\frac{1+f_{k}\left(x_{n}^{(k)}\right)}{f_{1}\left(x_{n-1}^{(1)}\right)}\right), \\
x_{n+1}^{(k)} & =f_{k}^{-1}\left(\frac{1+f_{1}\left(x_{n}^{(1)}\right)}{f_{2}\left(x_{n-1}^{(2)}\right)}\right), n \in \mathbb{N}_{0},
\end{aligned}
$$

where $k \in \mathbb{N} \backslash\{1\}$, and functions $f_{j}, j=\overline{1, k}$, are continuous on their domains; map the set $\mathbb{R} \backslash\{0\}$ onto itself and, for each fixed $j \in\{1, \ldots, k\}, f_{j}$ is simultaneously increasing or decreasing on the intervals $(-\infty, 0)$ and $(0,+\infty)$.

Then the following statements hold.

(a) If $k \neq \equiv 0(\bmod 5)$, then every well-defined solution of system (12) is periodic with period $5 k$.

(b) If $k \equiv 0(\bmod 5)$, then every well-defined solution of system (12) is periodic with period $k$.

Proof. From the conditions of the theorem, it follows that for each $j \in\{1, \ldots, k\}$, there is $f_{j}^{-1}$ which continuously map the set $\mathbb{R} \backslash\{0\}$ onto itself. Using the change of variables

$$
y_{n}^{(j)}=f_{j}\left(x_{n}^{(j)}\right), \quad j=\overline{1, k},
$$

system (12) is easily transformed into the next one

$$
y_{n+1}^{(1)}=\frac{1+y_{n}^{(2)}}{y_{n-1}^{(1)}}, \quad y_{n+1}^{(2)}=\frac{1+y_{n}^{(1)}}{y_{n-1}^{(2)}}
$$

for $k=2$,

$$
y_{n+1}^{(1)}=\frac{1+y_{n}^{(2)}}{y_{n-1}^{(3)}}, \quad y_{n+1}^{(2)}=\frac{1+y_{n}^{(3)}}{y_{n-1}^{(1)}}, \quad y_{n+1}^{(3)}=\frac{1+y_{n}^{(1)}}{y_{n-1}^{(2)}} \text {, }
$$

for $k=3$, and

$$
y_{n+1}^{(1)}=\frac{1+y_{n}^{(2)}}{y_{n-1}^{(3)}}, \quad y_{n+1}^{(2)}=\frac{1+y_{n}^{(3)}}{y_{n-1}^{(4)}}, \ldots, y_{n+1}^{(k)}=\frac{1+y_{n}^{(1)}}{y_{n-1}^{(2)}}
$$

for $k \geq 4$. In [4], it was proved that, if $k \neq \equiv 0(\bmod 5)$, then every well-defined solution of systems (14)-(16) is periodic 
with period $5 k$, and, if $k \equiv 0(\bmod 5)$, then every welldefined solution of systems (14)-(16) is periodic with period $k$. Using this along with the fact

$$
x_{n}^{(j)}=f_{j}^{-1}\left(y_{n}^{(j)}\right), \quad j=\overline{1, k}
$$

following from (13), the results in (a) and (b) follow.

The following theorem is proved in a similar way. Therefore, the proof will be omitted.

Theorem 2. Consider the following system of difference equations

$$
\begin{aligned}
x_{n+1}^{(1)} & =f_{1}^{-1}\left(\frac{1+f_{k}\left(x_{n}^{(k)}\right)}{f_{k-1}\left(x_{n-1}^{(k-1)}\right)}\right), \\
x_{n+1}^{(2)} & =f_{2}^{-1}\left(\frac{1+f_{1}\left(x_{n}^{(1)}\right)}{f_{k}\left(x_{n-1}^{(k)}\right)}\right), \\
& \vdots \\
x_{n+1}^{(k)} & =f_{k}^{-1}\left(\frac{1+f_{k-1}\left(x_{n}^{(k-1)}\right)}{f_{k-2}\left(x_{n-1}^{(k-2)}\right)}\right), \quad n \in \mathbb{N}_{0},
\end{aligned}
$$

where $k \in \mathbb{N} \backslash\{1\}$, and functions $f_{j}, j=\overline{1, k}$, are continuous on their domains; map the set $\mathbb{R} \backslash\{0\}$ onto itself and, for each fixed $j \in\{1, \ldots, k\}, f_{j}$ is simultaneously increasing or decreasing on the intervals $(-\infty, 0)$ and $(0,+\infty)$.

Then the following statements hold.

(a) If $k \neq \equiv 0(\bmod 5)$, then every well-defined solution of system (18) is periodic with period $5 k$.

(b) If $k \equiv 0(\bmod 5)$, then every well-defined solution of system (18) is periodic with period $k$.

Now, we show that all the results on the periodicity of the solutions of systems (3)-(6) in [19] follow from Theorems 1 and 2 .

Corollary 3. Systems of difference equations (3)-(6) are all periodic with period ten.

Proof. For the systems of difference equations (3)-(6), we use the following changes of variables, respectively:

$$
\begin{array}{ll}
x_{n}=\frac{1}{u_{n}}, & y_{n}=\frac{1}{v_{n}}, \quad n \in \mathbb{N}_{0} ; \\
x_{n}=-\frac{1}{u_{n}}, & y_{n}=-\frac{1}{v_{n}}, \quad n \in \mathbb{N}_{0} ; \\
x_{n}=-\frac{1}{u_{n}}, & y_{n}=\frac{1}{v_{n}}, \quad n \in \mathbb{N}_{0} ; \\
x_{n}=\frac{1}{u_{n}}, & y_{n}=-\frac{1}{v_{n}}, \quad n \in \mathbb{N}_{0} .
\end{array}
$$

By using them, systems (3)-(6) are transformed into system (14). By applying Theorem 1(a), ten-periodicity of all welldefined solutions of system (14) follows, from which tenperiodicity of all well-defined solutions of systems (3)-(6) follows.

The following four examples are natural extensions of systems (3)-(6).

Example 4. If we use the following functions:

$$
f_{1}(x)=\frac{1}{x^{l}}, \quad f_{2}(x)=\frac{1}{x^{m}},
$$

where $l$ and $m$ are odd integers, we see that all the conditions in Theorem 1 are applied with $k=2$, so by using the theorem we obtain that the system of difference equations

$$
\begin{aligned}
& x_{n+1}=\frac{1}{x_{n-1}}\left(\frac{y_{n}^{m}}{1+y_{n}^{m}}\right)^{1 / l}, \\
& y_{n+1}=\frac{1}{y_{n-1}}\left(\frac{x_{n}^{l}}{1+x_{n}^{l}}\right)^{1 / m},
\end{aligned}
$$

$n \in \mathbb{N}_{0}$, is also periodic with period ten.

Example 5. For

$$
f_{1}(x)=-\frac{1}{x^{l}}, \quad f_{2}(x)=-\frac{1}{x^{m}},
$$

where $l$ and $m$ are also odd integers, all the conditions of Theorem 1 are again satisfied with $k=2$. Using the theorem, it follows that the system

$$
\begin{aligned}
& x_{n+1}=\frac{1}{x_{n-1}}\left(\frac{y_{n}^{m}}{-1+y_{n}^{m}}\right)^{1 / l}, \\
& y_{n+1}=\frac{1}{y_{n-1}}\left(\frac{x_{n}^{l}}{-1+x_{n}^{l}}\right)^{1 / m},
\end{aligned}
$$

$n \in \mathbb{N}_{0}$, is ten-periodic.

Example 6. For

$$
f_{1}(x)=\frac{1}{x^{l}}, \quad f_{2}(x)=-\frac{1}{x^{m}},
$$

where $l$ and $m$ are odd integers, all the conditions of Theorem 1 are also satisfied with $k=2$. Applying the theorem, it follows that the system

$$
\begin{aligned}
x_{n+1} & =\frac{1}{x_{n-1}}\left(\frac{y_{n}^{m}}{-1+y_{n}^{m}}\right)^{1 / l}, \\
y_{n+1} & =\frac{1}{y_{n-1}}\left(\frac{x_{n}^{l}}{1+x_{n}^{l}}\right)^{1 / m},
\end{aligned}
$$

$n \in \mathbb{N}_{0}$, is ten-periodic.

Example 7. Finally, for

$$
f_{1}(x)=-\frac{1}{x^{l}}, \quad f_{2}(x)=\frac{1}{x^{m}},
$$


where $l$ and $m$ are odd integers and applying Theorem 1 with $k=2$, we get that the system

$$
\begin{aligned}
& x_{n+1}=\frac{1}{x_{n-1}}\left(\frac{y_{n}^{m}}{1+y_{n}^{m}}\right)^{1 / l}, \\
& y_{n+1}=\frac{1}{y_{n-1}}\left(\frac{x_{n}^{l}}{-1+x_{n}^{l}}\right)^{1 / m},
\end{aligned}
$$

$n \in \mathbb{N}_{0}$, is ten-periodic.

The main results in [4] can be relatively easily extended to a very general situation, which have been noticed by Iričanin and Stević soon after publishing [4], and later also proved by several other authors. Namely, the following result holds (see, e.g., [1]).

Theorem 8. Assume that the following difference equation

$$
x_{n}=f\left(x_{n-1}, \ldots, x_{n-k}\right), \quad n \in \mathbb{N}_{0} \text {, }
$$

is periodic with period $p$.

Then the following system of difference equations

$$
\begin{array}{r}
x_{n}^{(i)}=f\left(x_{n-1}^{(\sigma(i))}, x_{n-2}^{\left(\sigma^{[2]}(i)\right)}, \ldots, x_{n-k}^{\left(\sigma^{[k]}(i)\right)}\right), \\
i=\overline{1, l}, \quad n \in \mathbb{N}_{0},
\end{array}
$$

where $\sigma(i)=i+1$, for $1 \leq i \leq l-1, \sigma(l)=1$ and $\sigma^{[j]}(i)=$ $\sigma\left(\sigma^{[j-1]}(i)\right), j=\overline{1, k}$, and $\sigma^{[0]}(i)=i, i=\overline{1, l}$, is periodic with period $1 \mathrm{~cm}(p, l)$ (the least common multiple of numbers $p$ and $l$ ).

Theorem 8 can be used in constructing numerous periodic cyclic systems of difference equations based on scalar periodic difference equations, which, with some changes of variables, give some other periodic cyclic systems of difference equations.

\section{Periodicity of Positive Solutions of System (11)}

In this section, we study positive solutions of system (11). By $\operatorname{gcd}(s, k)$, we denote the greatest common divisor of natural numbers $s$ and $k$.

Theorem 9. Consider system (11). Assume that $s, k \in \mathbb{N}$, and $\left(A_{n}\right)_{n \in \mathbb{N}_{0}}$ and $\left(B_{n}\right)_{n \in \mathbb{N}_{0}}$ are positive $k \operatorname{gcd}(s, k)$-periodic sequences. Then every positive solution of system (11) is periodic with, not necessarily prime, period

$$
p=2 k \operatorname{gcd}(s, k) \text {. }
$$

Proof. Let $r=\operatorname{gcd}(s, k)$. Then we have that $s=r s_{1}$ and $k=r k_{1}$ for some $s_{1}, k_{1} \in \mathbb{N}$ such that

$$
\operatorname{gcd}\left(s_{1}, k_{1}\right)=1
$$

Since every $n \in \mathbb{N}_{0}$ can be written as $n=m r+i$, for some $m \in \mathbb{N}_{0}$ and $i=\overline{0, r-1}$, system (11) becomes

$$
\begin{aligned}
& x_{m r+i}=\max \left\{\frac{A_{m r+i}}{x_{r\left(m-s_{1}\right)+i}}, y_{r\left(m-k_{1}\right)+i}\right\}, \\
& y_{m r+i}=\max \left\{\frac{B_{m r+i}}{y_{r\left(m-s_{1}\right)+i}}, x_{r\left(m-k_{1}\right)+i}\right\},
\end{aligned}
$$

for every $m \in \mathbb{N}_{0}$ and $i=\overline{0, r-1}$.

Using the next change of variables

$$
x_{t}^{(i)}=x_{t r+i}, \quad y_{t}^{(i)}=y_{t r+i}
$$

where $t \geq-\max \left\{s_{1}, k_{1}\right\}, i=\overline{0, r-1}$, in (32), we have that $\left(x_{t}^{(i)}\right)_{t \geq-\max \left\{s_{1}, k_{1}\right\}},\left(y_{t}^{(i)}\right)_{t \geq-\max \left\{s_{1}, k_{1}\right\}}, i=\overline{0, r-1}$, are $r$ independent solutions of the next systems

$$
x_{t}=\max \left\{\frac{A_{t r+i}}{x_{t-s_{1}}}, y_{t-k_{1}}\right\}, \quad y_{t}=\max \left\{\frac{B_{t r+i}}{y_{t-s_{1}}}, x_{t-k_{1}}\right\} \text {, }
$$

which are systems of the form in (11) with $s_{1}$ and $k_{1}$ instead of $s$ and $k$, and where the sequences $\left(A_{t r+i}\right)_{t \in \mathbb{N}_{0}}$ and $\left(B_{t r+i}\right)_{t \in \mathbb{N}_{0}}$, $i=\overline{1, r}$, are $k$-periodic.

Hence, it is enough to prove the theorem when $\operatorname{gcd}(s, k)=$ 1 and the sequences $\left(A_{n}\right)_{n \in \mathbb{N}_{0}}$, and $\left(B_{n}\right)_{n \in \mathbb{N}_{0}}$ are positive $k$ periodic.

Now note that from the equations in (11), we have that

$$
x_{n} \geq y_{n-k}, \quad y_{n} \geq x_{n-k}, \quad \text { for } n \in \mathbb{N}_{0} .
$$

Further, by using the equations in (11), we also get

$$
\begin{aligned}
& x_{n}=\max \left\{\frac{A_{n}}{x_{n-s}}, y_{n-k}\right\}=\max \left\{\frac{A_{n}}{x_{n-s}}, \frac{B_{n-k}}{y_{n-k-s}}, x_{n-2 k}\right\}, \\
& y_{n}=\max \left\{\frac{B_{n}}{y_{n-s}}, x_{n-k}\right\}=\max \left\{\frac{B_{n}}{y_{n-s}}, \frac{A_{n-k}}{x_{n-k-s}}, y_{n-2 k}\right\},
\end{aligned}
$$

for $n \geq k$

Using relations (36), we get

$$
\begin{aligned}
x_{n} & =\max \left\{\frac{A_{n}}{x_{n-s}}, \frac{B_{n-k}}{y_{n-k-s}}, x_{n-2 k}\right\} \\
& =\max \left\{\frac{A_{n}}{x_{n-s}}, \frac{B_{n-k}}{y_{n-k-s}}, \frac{A_{n-2 k}}{x_{n-2 k-s}}, \frac{B_{n-3 k}}{y_{n-3 k-s}}, x_{n-4 k}\right\}, \\
y_{n} & =\max \left\{\frac{B_{n}}{y_{n-s}}, \frac{A_{n-k}}{x_{n-k-s}}, y_{n-2 k}\right\} \\
& =\max \left\{\frac{B_{n}}{y_{n-s}}, \frac{A_{n-k}}{x_{n-k-s}}, \frac{B_{n-2 k}}{y_{n-2 k-s}}, \frac{A_{n-3 k}}{x_{n-3 k-s}}, y_{n-4 k}\right\},
\end{aligned}
$$

for $n \geq 3 k$.

Now, note that, from the inequalities in (35), we have that

$$
x_{n} \geq x_{n-2 k}, \quad y_{n} \geq y_{n-2 k}, \quad \text { for } n \geq k .
$$


Using (38) and $k$-periodicity of the sequences $A_{n}$ and $B_{n}$, we obtain

$$
\begin{gathered}
\frac{A_{n}}{x_{n-s}}=\frac{A_{n-2 k}}{x_{n-s}} \leq \frac{A_{n-2 k}}{x_{n-2 k-s}}, \\
\frac{B_{n-k}}{y_{n-k-s}}=\frac{B_{n-3 k}}{y_{n-k-s}} \leq \frac{B_{n-3 k}}{y_{n-3 k-s}}, \\
\frac{B_{n}}{y_{n-s}}=\frac{B_{n-2 k}}{y_{n-s}} \leq \frac{B_{n-2 k}}{y_{n-2 k-s}}, \\
\frac{A_{n-k}}{x_{n-k-s}}=\frac{A_{n-3 k}}{x_{n-k-s}} \leq \frac{A_{n-3 k}}{x_{n-3 k-s}} .
\end{gathered}
$$

Employing (39) into (37), we get

$$
\begin{aligned}
& x_{n}=\max \left\{\frac{A_{n-2 k}}{x_{n-2 k-s}}, \frac{B_{n-3 k}}{y_{n-3 k-s}}, x_{n-4 k}\right\}=x_{n-2 k}, \\
& y_{n}=\max \left\{\frac{B_{n-2 k}}{y_{n-2 k-s}}, \frac{A_{n-3 k}}{x_{n-3 k-s}}, y_{n-4 k}\right\}=y_{n-2 k},
\end{aligned}
$$

from which it follows that in this case the solutions of system (11) are $2 k$-periodic. From all the above, the theorem follows.

By a slight modification of the proof of Theorem 9, the next result can be proved. We omit the proof.

Theorem 10. Consider the following system of difference equations

$$
\begin{gathered}
x_{n}=\max \left\{\frac{A_{n}}{x_{n-s}}, y_{n-k}\right\}, \quad y_{n}=\max \left\{\frac{B_{n}}{y_{n-s}}, z_{n-k}\right\}, \\
z_{n}=\max \left\{\frac{C_{n}}{z_{n-s}}, x_{n-k}\right\}, \quad n \in \mathbb{N}_{0},
\end{gathered}
$$

where $s, k \in \mathbb{N}$, and $\left(A_{n}\right)_{n \in \mathbb{N}_{0}},\left(B_{n}\right)_{n \in \mathbb{N}_{0}}$, and $\left(C_{n}\right)_{n \in \mathbb{N}_{0}}$ are positive $k \operatorname{gcd}(s, k)$-periodic sequences. Then, every positive solution of system (41) is periodic with, not necessarily prime, period

$$
p=3 k \operatorname{gcd}(s, k) \text {. }
$$

\section{Conflict of Interests}

The authors declare that there is no conflict of interests regarding the publication of this paper.

\section{Acknowledgments}

This work was funded by the Deanship of Scientific Research (DSR), King Abdulaziz University, under Grant no. (11130/1433 HiCi). The authors, therefore, acknowledge technical and financial support of KAU.

\section{References}

[1] G. Bastien and M. Rogalski, "Global behaviour of solutions of cyclic systems of $q$ order 2 or 3 generalized Lyness' difference equations and of other more general equations of higher order," Journal of Difference Equations and Applications, vol. 17, no. 11, pp. 1651-1672, 2011.

[2] E. M. Elsayed, B. Iričanin, and S. Stević, "On the max-type equation $x_{n+1}=\max \left\{A_{n} / x_{n}, x_{n-1}\right\}$," Ars Combinatoria, vol. 95, pp. 187-192, 2010.

[3] E. A. Grove and G. Ladas, Periodicities in Nonlinear Difference Equations, vol. 4 of Advances in Discrete Mathematics and Applications, Chapman \& Hall, CRC Press, Boca Raton, Fla, USA, 2005.

[4] B. D. Iričanin and S. Stević, "Some systems of nonlinear difference equations of higher order with periodic solutions," Dynamics of Continuous, Discrete \& Impulsive Systems A, vol. 13, no. 3-4, pp. 499-507, 2006.

[5] B. D. Iričanin and N. Touafek, "On a second-order max-type system of difference equations," Indian Journal of Mathematics, vol. 54, no. 1, pp. 119-142, 2012.

[6] C. M. Kent, M. Kustesky, A. Q. Nguyen, and B. V. Nguyen, "Eventually periodic solutions of $x_{n+1}=\max \left\{A_{n} / x_{n}, B_{n} / x_{n-1}\right\}$ when the parameters are two cycles", Dynamics of Continuous, Discrete \& Impulsive Systems A, vol. 10, no. 1-3, pp. 33-49, 2003.

[7] C. M. Kent and M. A. Radin, "On the boundedness nature of positive solutions of the difference equation $x_{n+1}=$ $\max \left\{A_{n} / x_{n}, B_{n} / x_{n-1}\right\}$ with periodic parameters," Dynamics of Continuous, Discrete \& Impulsive Systems B, supplement, pp. 11$15,2003$.

[8] W. Kosmala, "A period 5 difference equation," International Journal of Nonlinear Analysis and Applications, vol. 2, no. 1, pp. 82-84, 2011.

[9] G. Papaschinopoulos and C. J. Schinas, "On the behavior of the solutions of a system of two nonlinear difference equations," Communications on Applied Nonlinear Analysis, vol. 5, no. 2, pp. 47-59, 1998.

[10] G. Papaschinopoulos and C. J. Schinas, "Invariants for systems of two nonlinear difference equations," Differential Equations and Dynamical Systems, vol. 7, no. 2, pp. 181-196, 1999.

[11] G. Papaschinopoulos, J. Schinas, and V. Hatzifilippidis, "Global behavior of the solutions of a max-equation and of a system of two max-equations," Journal of Computational Analysis and Applications, vol. 5, no. 2, pp. 237-254, 2003.

[12] S. Stević, "A global convergence results with applications to periodic solutions," Indian Journal of Pure and Applied Mathematics, vol. 33, no. 1, pp. 45-53, 2002.

[13] S. Stević, "On the recursive sequence $x_{n+1}=\alpha_{n}+x_{n-1} / x_{n}$ II," Dynamics of Continuous, Discrete \& Impulsive Systems A, vol. 10, no. 6, pp. 911-916, 2003.

[14] S. Stević, "A short proof of the Cushing-Henson conjecture," Discrete Dynamics in Nature and Society, vol. 2006, Article ID 37264, 5 pages, 2006.

[15] S. Stević, "Global stability of a difference equation with maximum," Applied Mathematics and Computation, vol. 210, no. 2, pp. 525-529, 2009.

[16] S. Stević, "Periodicity of max difference equations," Utilitas Mathematica, vol. 83, pp. 69-71, 2010.

[17] S. Stević, "Periodicity of a class of nonautonomous max-type difference equations," Applied Mathematics and Computation, vol. 217, no. 23, pp. 9562-9566, 2011. 
[18] S. Stević, "On some periodic systems of max-type difference equations," Applied Mathematics and Computation, vol. 218, no. 23, pp. 11483-11487, 2012.

[19] N. Touafek and E. M. Elsayed, "On the periodicity of some systems of nonlinear difference equations," Bulletin Mathématique de la Société des Sciences Mathématiques de Roumanie. Nouvelle Série, vol. 55, no. 2, pp. 217-224, 2012.

[20] H. Levy and F. Lessman, Finite Difference Equations, Macmillan, New York, NY, USA, 1961.

[21] R. C. Lyness, "Notes 1581," The Mathematical Gazette, vol. 26, p. 62, 1942.

[22] R. C. Lyness, "Notes 1847," The Mathematical Gazette, vol. 29, p. 231, 1945.

[23] R. C. Lyness, "Notes 2952," The Mathematical Gazette, vol. 45, p. 201, 1961. 


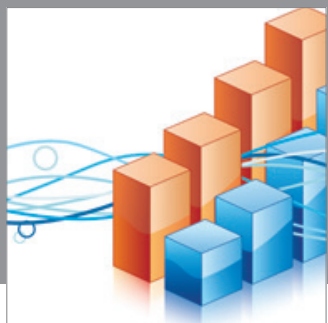

Advances in

Operations Research

mansans

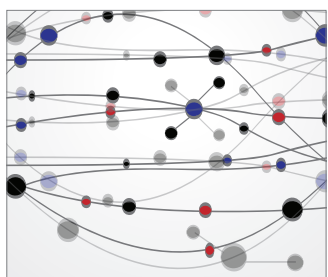

The Scientific World Journal
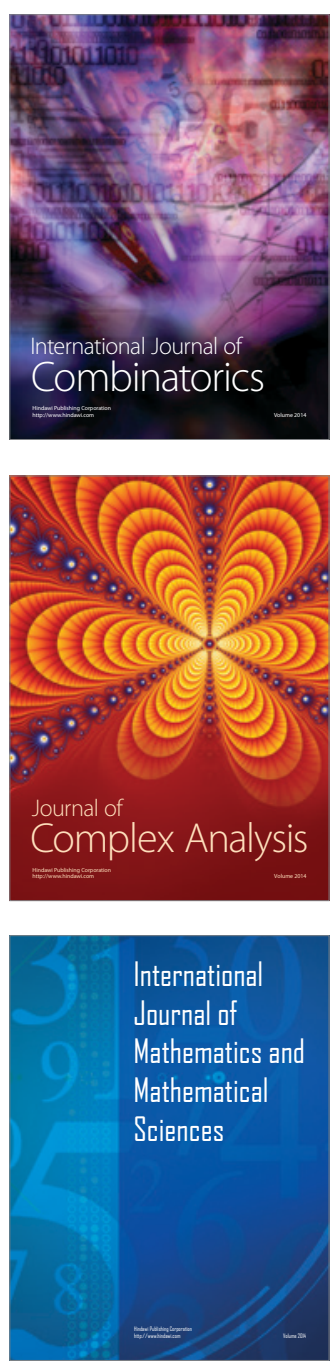
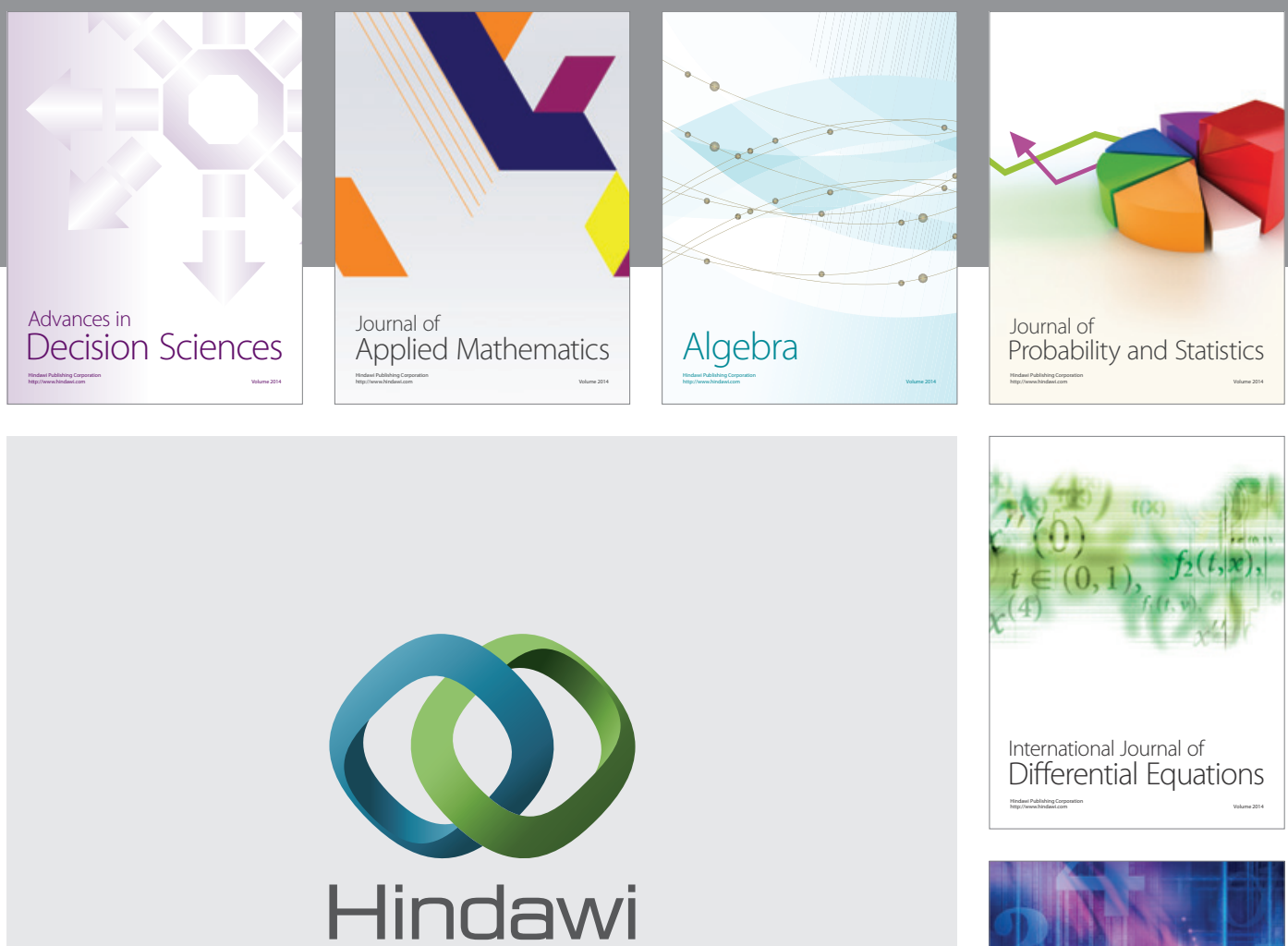

Submit your manuscripts at http://www.hindawi.com
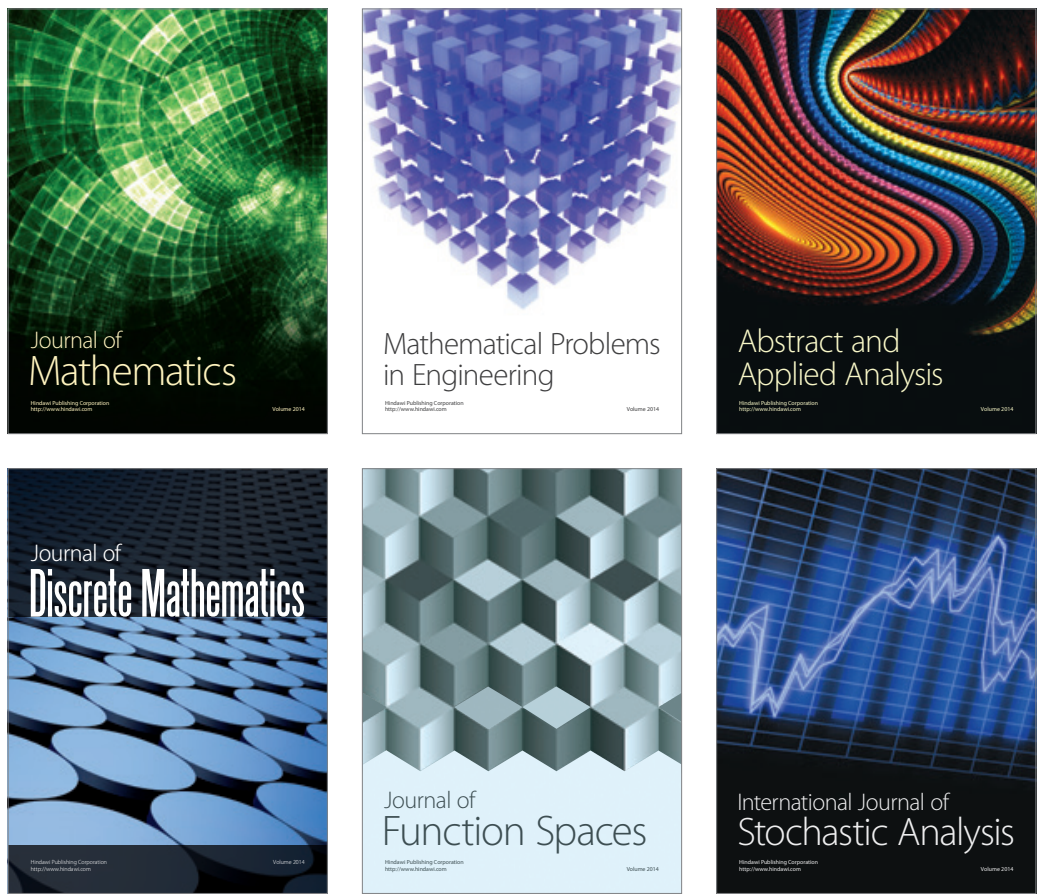

Journal of

Function Spaces

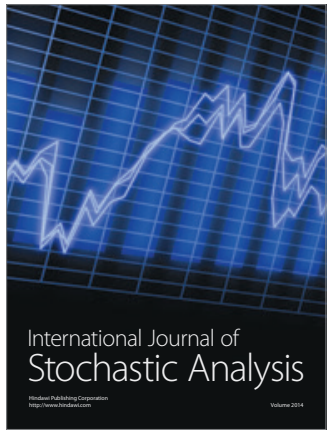

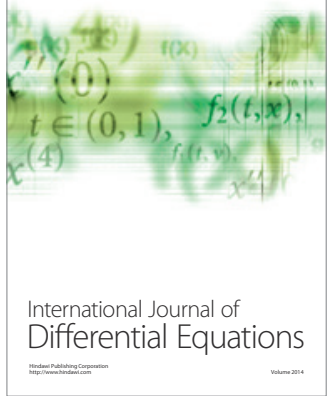
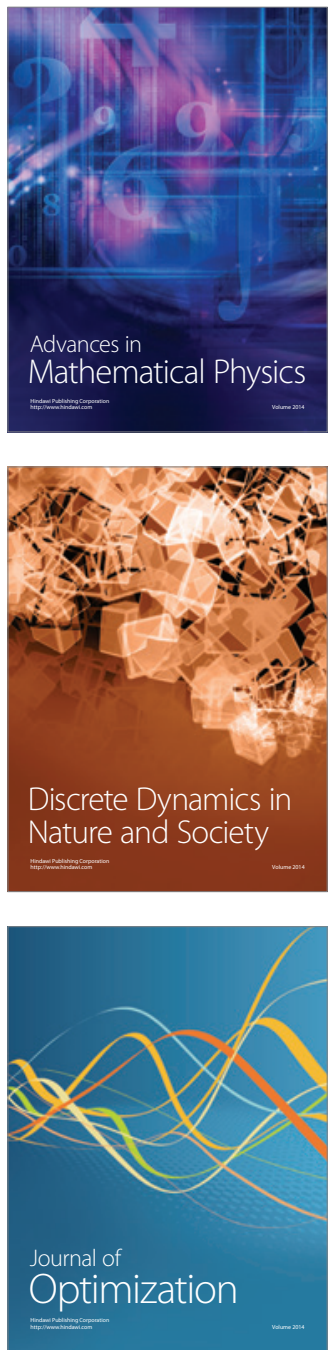